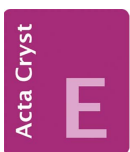

CRYSTALLOGRAPHIC COMMUNICATIONS

ISSN 2056-9890

Received 22 May 2020

Accepted 17 June 2020

Edited by B. Therrien, University of Neuchâtel, Switzerland

Keywords: crystal structure; copper corrosion; equipment failure; posnjakite; hydrogen bonding.

CCDC reference: 2010348

Supporting information: this article has supporting information at journals.iucr.org/e

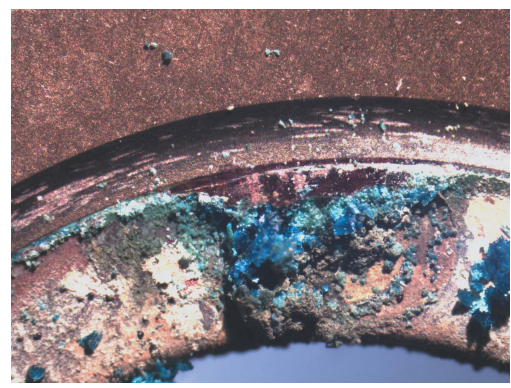

\section{Crystal structure of posnjakite formed in the first crystal water-cooling line of the ANSTO Melbourne Australian Synchrotron MX1 Double Crystal Monochromator}

\author{
Stuart Mills, ${ }^{a}$ Jun Aishima, ${ }^{\text {b,c }}$ David Aragao, ${ }^{\text {c,d }}$ Tom Tudor Caradoc-Davies, ${ }^{c}$ \\ Nathan Cowieson, ${ }^{c, d}$ Christine L. Gee, ${ }^{\mathrm{c}, \mathrm{e}}$ Daniel Ericsson, ${ }^{\mathrm{c}}$ Stephen Harrop, \\ Santosh Panjikar, ${ }^{\mathrm{C}}$ Kate Mary Louise Smith, ${ }^{\mathrm{c}}$ Alan Riboldi-Tunnicliffe, ${ }^{\mathrm{c}}$ Rachel \\ Williamson $^{\mathrm{c}}$ and Jason Roy Price ${ }^{\mathrm{c} *}$
}

\footnotetext{
${ }^{\mathbf{a}}$ Geosciences, Museum Victoria, GPO Box 666, Melbourne, Victoria, 3001, Australia, ${ }^{\mathbf{b}}$ Brookhaven National Laboratory, 743 Brookhaven Avenue, Upton, NY, USA, 'Australian Synchrotron, ANSTO - Melbourne, 800 Blackburn Rd, Clayton, VIC, 3168, Australia, 'Diamond Light Source, Diamond Light Source Ltd, Didcot, Oxfordshire, OX11 ODE, UK, and e Department of Molecular and Cell Biology, University of California, Berkeley, CA 94720, USA. *Correspondence e-mail: jason.price@ansto.gov.au
}

Exceptionally large crystals of posnjakite, $\mathrm{Cu}_{4} \mathrm{SO}_{4}(\mathrm{OH})_{6}\left(\mathrm{H}_{2} \mathrm{O}\right)$, formed during corrosion of a Swagelock(tm) Snubber copper gasket within the MX1 beamline at the ANSTO-Melbourne, Australian Synchrotron. The crystal structure was solved using synchrotron radiation to $R_{1}=0.029$ and revealed a structure based upon $\left[\mathrm{Cu}_{4}(\mathrm{OH})_{6}\left(\mathrm{H}_{2} \mathrm{O}\right) \mathrm{O}\right]$ sheets, which contain Jahn-Teller-distorted $\mathrm{Cu}$ octahedra. The sulfate tetrahedra are bonded to one side of the sheet via corner sharing and linked to successive sheets via extensive hydrogen bonds. The sulfate tetrahedra are split and rotated, which enables additional hydrogen bonds.

\section{Chemical context}

The MX1 beamline at the ANSTO-Melbourne Australian Synchrotron has been operating since 2007 (Cowieson et al., 2015). The vacuum vessel for the double crystal monochromator suffered a loss of vacuum in July 2019. Investigation lead to the discovery of a pinhole leak in the watercooling system for the first crystal. On disassembly, there was the discovery of corrosion, oxidation and a crust of crystal aggregates deposited on the Swagelock ${ }^{(\mathrm{tm})}$ Snubber copper gasket within the connection. The coolant for the system is reverse osmosis ( $\mathrm{RO})$ water, kept at constant temperature of $20^{\circ} \mathrm{C}$ by a Huber Chiller (CC-K6, Pilot ONE) and previously had Aqua-Stabil (Jubalo $\mathrm{GmbH}$ ) added to prevent microbial and fungal growth. The lines are a combination of plastic and stainless steel, with water passing through an oxygen-free copper block into which the Silicon 111 first crystal is clamped with a layer of indium between the silicon crystal and the copper cooling block. The Aqua-Stabil was removed from service following a review of the environmental risks from chemicals in use. The $\mathrm{pH}$-neutral $\mathrm{RO}$ water has been used as a coolant since 2015 . In order to understand how the crystalline material was formed, the MX1 beamline was used after repairs were enacted.

A thin film of red copper oxide (cuprite) coats the surface of the gasket, and on top of that sits a mat of crystals up to $0.5 \mathrm{~cm}$ thick (Fig. 1). Above the cuprite coating a pale-blue X-ray 


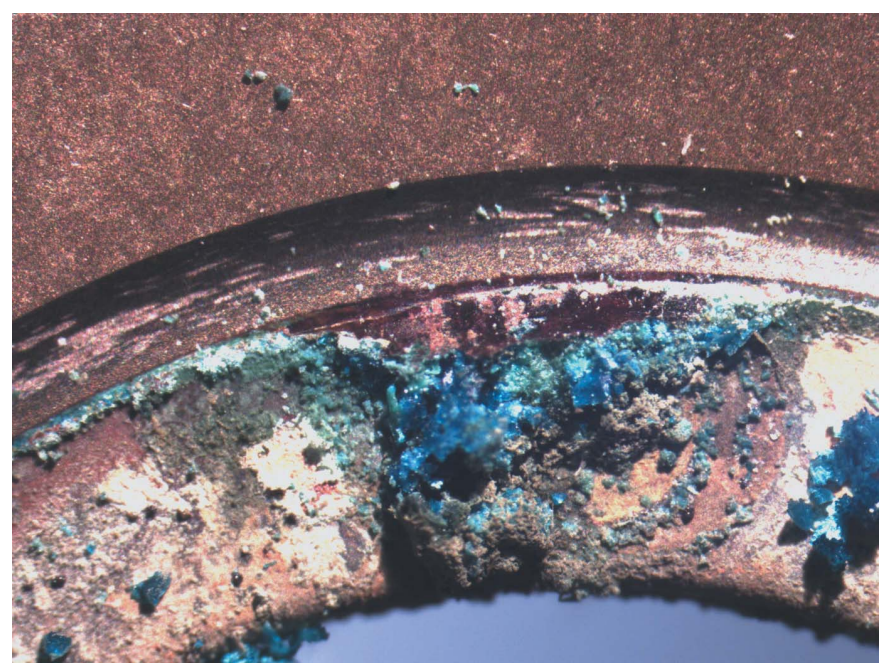

Figure 1

The copper Swagelok Snubber gasket from the MX1 cooling line for the first crystal in the double crystal monochromater showing crystalline deposits.

amorphous phase is observed, and perched on this phase sits exceptionally well-crystallized dark-blue crystals of posnjakite. The crystals of posnjakite are tabular on (101) with maximum dimension of $\sim 0.2 \times 0.2 \times 0.2 \mathrm{~mm}$. On some crystals, dark-green tips are observed where the crystals are transforming to brochantite.

\section{Formation of the crystals}

The corrosion of copper is of great interest in corrosion science, the arts and in the understanding of electrical apparatus failures. As is in our case, the copper corrosion has resulted in equipment failure. It has been seen that corrosive sulfur in oil has become a problem in electrical apparatus failures, where it is noted 'a number of failures of very large power transformers and shunt reactors associated with corrosive sulfur in electrical insulating mineral oils. Although the number of failures has been relatively small, perhaps 100 or so units, the assets lost have been substantial' (Griffin \& Lewand 2007). Although no sulfur is in the MX1 system, the copper gasket was produced using milling oils that have sulfurized fat as a component. Even though these gaskets are cleaned before use, it is apparent that some residue remained and that it contains enough sulfur to produce abundant mineralization if a pinhole leak exists.

The production of copper patinas in several outdoor conditions has been charted (Krätschmer et al. 2002) and it was shown that cuprite was formed immediately, followed by an amorphous copper sulfate over hours to weeks, posnjakite over weeks to months and finally brochantite over years. We see this entire assemblage on the MX1 gasket; however, brochantite is the least common of the phases. We can use this data to estimate that the leak was present for approximately a year before failure. What is interesting is that the formation in this moderately closed system has enhanced the formation of posnajkite, creating sub-millimetre-sized crystals. It is worth

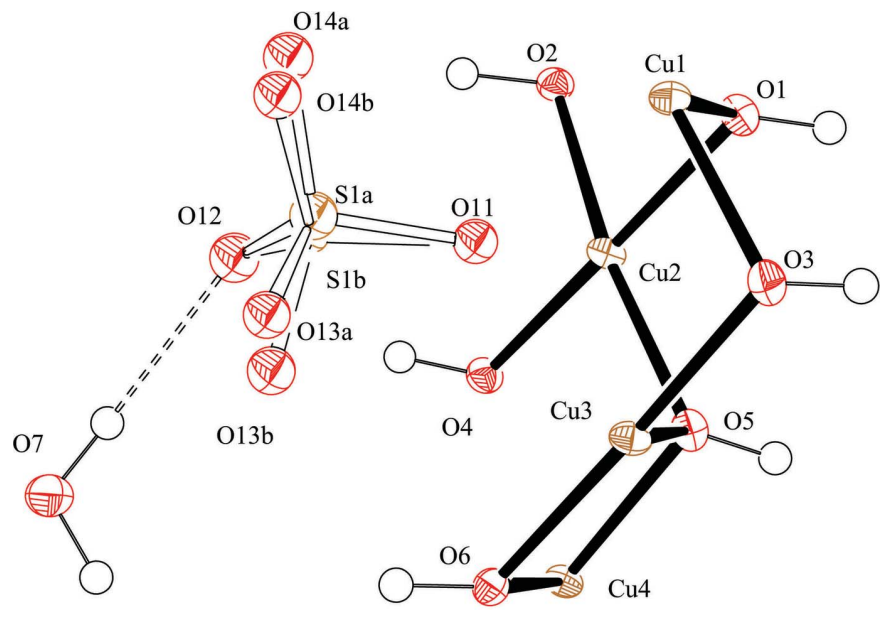

Figure 2

The asymmetric unit of posnjakite, the atomic numbering scheme is shown, and displacement ellipsoids are drawn at the $50 \%$ probability level.

noting that posnajkite is metastable with respect to brochantite (Zittlau et al., 2013); however, it is also common especially in geological systems that the crystalline phase that may form in a system that is not the most stable one, but very frequently metastable with a simpler structure (Krivovichev, 2017).

\section{Structural commentary and supramolecular features}

Mellini \& Merlino (1979) published the original structure of posnjakite, but with one $\mathrm{H}$ atom missing and limited thermal parameters. Our dataset has enabled the location of all $\mathrm{H}$ atoms from the difference map as well as an anisotropic model of all non-hydrogen atoms, except for the disordered sulfate. The posnjakite structure is based upon $\left[\mathrm{Cu}_{4}(\mathrm{OH})_{6}\left(\mathrm{H}_{2} \mathrm{O}\right) \mathrm{O}\right]$ sheets, which contain Jahn-Teller-distorted $\mathrm{Cu}$ octahedra. The average bond lengths for the octahedra are 2.08, 2.07, 2.11 and 2.11 for $\mathrm{Cu} 1-4$, respectively. These octahedra are more regular

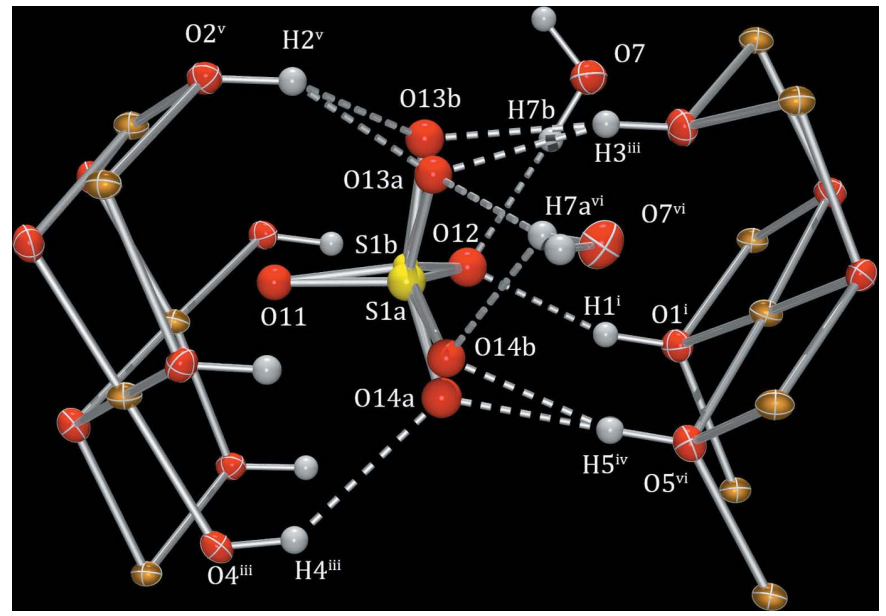

Figure 3

The complex arrangement of hydrogen bonds surrounding the disordered sulfate anion (ORTEP-3; Farrugia, 2012). Symmetry codes: (i) $x+\frac{1}{2}, 1-y$, $z-\frac{3}{2}$; (ii) $x+\frac{1}{2}, 2-y, z+\frac{1}{2}$; (iii) $x+\frac{1}{2}, 2-y, z-\frac{1}{2}$; (iv) $x+1, y, z$; (v) $x, y+1$, $z ;\left(\right.$ vi) $x+\frac{1}{2}, 2-y, z+\frac{1}{2}$. 
Table 1

Hydrogen-bond geometry $\left(\AA,^{\circ}\right)$.

\begin{tabular}{lllll}
\hline$D-\mathrm{H} \cdots A$ & $D-\mathrm{H}$ & $\mathrm{H} \cdots A$ & $D \cdots A$ & $D-\mathrm{H} \cdots A$ \\
\hline $\mathrm{O} 1-\mathrm{H} 1 \cdots \mathrm{O} 12^{\mathrm{i}}$ & $0.87(6)$ & $1.86(6)$ & $2.693(9)$ & $160(7)$ \\
$\mathrm{O} 7-\mathrm{H} 7 B \cdots \mathrm{O} 12$ & $0.87(3)$ & $2.02(4)$ & $2.844(7)$ & $158(7)$ \\
$\mathrm{O} 2-\mathrm{H} 2 \cdots \mathrm{O} 13 A^{\text {ii }}$ & $0.91(6)$ & $1.95(6)$ & $2.746(9)$ & $144(7)$ \\
$\mathrm{O} 2-\mathrm{H} 2 \cdots \mathrm{O} 13 B^{\text {ii }}$ & $0.91(6)$ & $1.96(7)$ & $2.731(18)$ & $141(7)$ \\
$\mathrm{O} 3-\mathrm{H} 3 \cdots \mathrm{O} 13 A^{\text {iii }}$ & $0.83(6)$ & $1.96(6)$ & $2.757(10)$ & $161(9)$ \\
$\mathrm{O} 3-\mathrm{H} 3 \cdots \mathrm{O} 13 B^{\text {iii }}$ & $0.83(6)$ & $1.99(7)$ & $2.799(19)$ & $164(9)$ \\
$\mathrm{O} 7-\mathrm{H} 7 A \cdots \mathrm{O} 13 A^{\text {iv }}$ & $0.89(3)$ & $2.13(3)$ & $2.995(10)$ & $167(7)$ \\
$\mathrm{O} 4-\mathrm{H} 4 \cdots \mathrm{O} 14 A^{\mathrm{v}}$ & $0.86(6)$ & $2.22(7)$ & $2.929(9)$ & $141(7)$ \\
$\mathrm{O} 4-\mathrm{H} 4 \cdots \mathrm{O} 14 B^{\text {v }}$ & $0.86(6)$ & $2.57(7)$ & $3.239(18)$ & $136(7)$ \\
$\mathrm{O} 5-\mathrm{H} 5 \cdots \mathrm{O} 14 A^{\text {vi }}$ & $0.87(6)$ & $1.86(6)$ & $2.727(9)$ & $177(8)$ \\
$\mathrm{O} 5-\mathrm{H} 5 \cdots \mathrm{O} 14 B^{\text {vi }}$ & $0.87(6)$ & $1.93(6)$ & $2.763(17)$ & $162(7)$ \\
O6-H6 $\cdots 13 B$ & $0.86(6)$ & $2.45(8)$ & $2.98(2)$ & $120(7)$ \\
O6-H6 $\cdots \mathrm{O} 14 B^{\text {iv }}$ & $0.86(6)$ & $2.47(7)$ & $3.211(17)$ & $145(7)$ \\
$\mathrm{O} 7-\mathrm{H} 7 A \cdots \mathrm{O} 13 B^{\text {iv }}$ & $0.89(3)$ & $2.64(4)$ & $3.489(18)$ & $161(7)$ \\
$\mathrm{O} 7-\mathrm{H} 7 A \cdots \mathrm{O} 14 B^{\text {iv }}$ & $0.89(3)$ & $2.31(6)$ & $3.034(19)$ & $139(7)$ \\
\hline & & & &
\end{tabular}

Symmetry codes: (i) $x-\frac{1}{2},-y+1, z+\frac{1}{2}$; (ii) $x, y-1, z$; (iii) $x-\frac{1}{2},-y+2, z+\frac{1}{2}$; (iv) $x-\frac{1}{2},-y+2, z-\frac{1}{2}$; (v) $x-\frac{1}{2},-y+1, z-\frac{1}{2}$; (vi) $x-1, y, z$.

than the ones observed previously (Mellini \& Merlino, 1979) because of the higher quality dataset and lower temperature refinement, which places the water molecule $\sim 0.3 \AA$ closer to the $\mathrm{Cu}$ atoms. The sheet is also less undulating than reported for the previous refinement. The asymmetric unit for the current structure is shown in Fig. 2.

The sulfate tetrahedra are bonded to one side of the sheet via corner sharing and linked to successive sheets via extensive hydrogen bonds (Table 1). The main difference in this dataset is that the sulfate tetrahedra are split and rotated by $\sim 7^{\circ}$. This allows greater connectivity between the sulfate oxygen atoms and the hydroxyl atoms in the sheet (e.g. O14A-H4) as well as the water of solvation (O13A-H7A, Fig. 3$)$.

The tetrahedra were restrained to have the grand mean $<\mathrm{S}-\mathrm{O}>$ of $1.473 \AA$ reported (Hawthorne et al. 2000), and the atoms kept isotropic.

\section{Refinement}

Crystal data, data collection and structure refinement details are summarized in Table 2. All non-hydrogen atom sites in the asymmetric unit were modelled with anisotropic displacement parameters with exception of the partially occupancy atoms which were left isotropic. The disordered sulfate tetrahedra were restrained to have the grand mean $<\mathrm{S}-\mathrm{O}>$ of $1.473 \AA$ reported (Hawthorne et al. 2000), and the atoms kept isotropic. Hydrogen atoms were located in the difference map and their coordinates refined with group displacement parameters $U_{\text {iso }}(\mathrm{H})=1.5 U_{\text {eq }}(\mathrm{O})$. Twinning of the crystal was explored to examine if the apparent disorder of the sulfate anion was from a twin component. An inversion twining was ruled out as the Flack (1983) parameter was 0.08 (4); further twinning was explored with TwinRotMat in the PLATON suite (Spek, 2020) with no twins found.

\section{Acknowledgements}

This research was undertaken in part using the MX1 beamline at the Australian Synchrotron, part of ANSTO.
Table 2

Experimental details.

\begin{tabular}{ll}
\hline Crystal data & \\
Chemical formula & $\mathrm{Cu}_{4}(\mathrm{SO})_{4}(\mathrm{OH})_{6}\left(\mathrm{H}_{2} \mathrm{O}\right)$ \\
$M_{\mathrm{r}}$ & 470.28 \\
Crystal system, space group & Monoclinic, $P n$ \\
Temperature $(\mathrm{K})$ & 100 \\
$a, b, c(\AA)$ & $7.8400(16), 6.3400(13), 9.768(2)$ \\
$\beta\left({ }^{\circ}\right.$ & $107.32(3)$ \\
$V\left(\AA^{3}\right)$ & $463.51(18)$ \\
$Z$ & 2 \\
Radiation type & Synchrotron, $\lambda=0.71074 \AA$ \\
$\mu\left(\mathrm{mm}^{-1}\right)$ & 9.33 \\
Crystal size (mm) & $0.15 \times 0.10 \times 0.05$ \\
& \\
Data collection & MX1 Beamline Australian Synchrotron \\
Diffractometer & Multi-scan $(S A D A B S ;$ Bruker, 2001) \\
Absorption correction & $0.517,0.746$ \\
$T_{\text {min }}, T_{\text {max }}$ & $5709,1763,1652$ \\
No. of measured, independent and & \\
$\quad$ observed $[I>2 \sigma(I)]$ reflections & \\
$R_{\text {int }}$ & 0.019 \\
(sin $\theta / \lambda)_{\text {max }}\left(\AA^{-1}\right)$ & 0.617 \\
& \\
Refinement & \\
$R\left[F^{2}>2 \sigma\left(F^{2}\right)\right], w R\left(F^{2}\right), S$ & $0.029,0.074,1.06$ \\
No. of reflections & 1763 \\
No. of parameters & 157 \\
No. of restraints & 19 \\
H-atom treatment & Only H-atom coordinates refined \\
$\Delta \rho_{\text {max }}, \Delta \rho_{\min }\left(\mathrm{e} \AA^{-3}\right)$ & $0.67,-0.92$ \\
Absolute structure & Flack $x$ determined using 745 quotients \\
& {$\left[\left(I^{+}\right)-\left(I^{-}\right)\right] /\left[\left(I^{+}\right)+\left(I^{-}\right)\right]$} \\
Absolute structure parameter & $0.128(16)$ \\
\hline &
\end{tabular}

Computer programs: AS QEGUI, XDS (Kabsch, 2010), SHELXT (Sheldrick, 2015a), SHELXL (Sheldrick, 2015b), shelXle (Hübschle et al., 2011), ORTEP-3 for Windows (Farrugia, 2012) and publCIF (Westrip, 2010).

\section{References}

Bruker (2001). Bruker AXS Inc., Madison, Wisconsin, USA.

Cowieson, N. P., Aragao, D., Clift, M., Ericsson, D. J., Gee, C., Harrop, S. J., Mudie, N., Panjikar, S., Price, J. R., Riboldi-Tunnicliffe, A., Williamson, R. \& Caradoc-Davies, T. (2015). J. Synchrotron Rad. 22, 187-190.

Farrugia, L. J. (2012). J. Appl. Cryst. 45, 849-854.

Flack, H. D. (1983). Acta Cryst. A39, 876-881.

Griffin, P. J. \& Lewand, L. R. (2007). Proceedings of the SeventyFourth Annual International Conference of Doble Clients. pp. $1-7$.

Hawthorne, F. C., Krivovichev, S. V. \& Burns, P. C. (2000). Rev. Mineral. Geochem. 40,1-112.

Hübschle, C. B., Sheldrick, G. M. \& Dittrich, B. (2011). J. Appl. Cryst. 44, 1281-1284.

Kabsch, W. (2010). Acta Cryst. D66, 125-132.

Krätschmer, A., Odnevall Wallinder, I. \& Leygraf, C. (2002). Corros. Sci. 44, 425-450.

Krivovichev, S. V. (2017). J. Geosci. pp. 79-85.

Mellini, M. \& Merlino, S. (1979). Z. Kristallogr. 149, 249-257.

Parsons, S., Flack, H. D. \& Wagner, T. (2013). Acta Cryst. B69, 249259.

Sheldrick, G. M. (2015a). Acta Cryst. A71, 3-8.

Sheldrick, G. M. (2015b). Acta Cryst. C71, 3-8.

Spek, A. L. (2020). Acta Cryst. E76, 1-11.

Westrip, S. P. (2010). J. Appl. Cryst. 43, 920-925.

Zittlau, A. H., Shi, Q., Boerio-Goates, J., Woodfield, B. F. \& Majzlan, J. (2013). Geochemistry, 73, 39-50. 


\section{supporting information}

Acta Cryst. (2020). E76, 1136-1138 [https://doi.org/10.1107/S2056989020008099]

Crystal structure of posnjakite formed in the first crystal water-cooling line of the ANSTO Melbourne Australian Synchrotron MX1 Double Crystal

\section{Monochromator}

Stuart Mills, Jun Aishima, David Aragao, Tom Tudor Caradoc-Davies, Nathan Cowieson,

Christine L. Gee, Daniel Ericsson, Stephen Harrop, Santosh Panjikar, Kate Mary Louise Smith, Alan Riboldi-Tunnicliffe, Rachel Williamson and Jason Roy Price

Computing details

Data collection: AS QEGUI; cell refinement: $X D S$ (Kabsch, 2010); data reduction: XDS (Kabsch, 2010)); program(s) used to solve structure: SHELXT (Sheldrick, 2015a); program(s) used to refine structure: SHELXL (Sheldrick, 2015b); molecular graphics: shelXle (Hübschle et al., 2011), ORTEP-3 for Windows (Farrugia, 2012); software used to prepare material for publication: publCIF (Westrip, 2010).

Poly[[hexa- $\mu$-hydroxido-tetracopper(II)] sulfate monohydrate]

Crystal data

$\mathrm{Cu}_{4}(\mathrm{SO})_{4}(\mathrm{OH})_{6}\left(\mathrm{H}_{2} \mathrm{O}\right)$

$F(000)=456$

$M_{r}=470.28$

$D_{\mathrm{x}}=3.370 \mathrm{Mg} \mathrm{m}^{-3}$

Monoclinic, $P n$

$a=7.8400(16) \AA$

$b=6.3400(13) \AA$

$c=9.768(2) \AA$

$\beta=107.32(3)^{\circ}$

$V=463.51(18) \AA^{3}$

$Z=2$

Synchrotron radiation, $\lambda=0.71074 \AA$

Cell parameters from 96 reflections

$\mu=9.33 \mathrm{~mm}^{-1}$

$T=100 \mathrm{~K}$

Plate, blue

$0.15 \times 0.10 \times 0.05 \mathrm{~mm}$

Data collection

MX1 Beamline Australian Synchrotron diffractometer

Radiation source: Double Crystal Monochromator

Silicon 111 scans

Absorption correction: multi-scan

(SADABS; Bruker, 2001)

$T_{\min }=0.517, T_{\max }=0.746$

5709 measured reflections

1763 independent reflections

1652 reflections with $I>2 \sigma(I)$

$R_{\text {int }}=0.019$

$\theta_{\max }=26.0^{\circ}, \theta_{\min }=2.9^{\circ}$

$h=-9 \rightarrow 9$

$k=-7 \rightarrow 7$

$l=-12 \rightarrow 11$

Refinement

Refinement on $F^{2}$

Least-squares matrix: full

$R\left[F^{2}>2 \sigma\left(F^{2}\right)\right]=0.029$

$w R\left(F^{2}\right)=0.074$

$S=1.06$

1763 reflections

157 parameters

19 restraints

Hydrogen site location: difference Fourier map

Only H-atom coordinates refined 
$w=1 /\left[\sigma^{2}\left(F_{\mathrm{o}}^{2}\right)+(0.0447 P)^{2}\right]$

where $P=\left(F_{\mathrm{o}}^{2}+2 F_{\mathrm{c}}^{2}\right) / 3$

$(\Delta / \sigma)_{\max }<0.001$

$\Delta \rho_{\max }=0.67$ e $\AA^{-3}$

$\Delta \rho_{\min }=-0.92$ e $\AA^{-3}$
Absolute structure: Flack $x$ determined using 745 quotients $\left[\left(I^{+}\right)-(I)\right] /\left[\left(I^{+}\right)+(I)\right]$ (Parsons et al., 2013)

Absolute structure parameter: 0.128 (16)

Special details

Geometry. All esds (except the esd in the dihedral angle between two 1.s. planes) are estimated using the full covariance matrix. The cell esds are taken into account individually in the estimation of esds in distances, angles and torsion angles; correlations between esds in cell parameters are only used when they are defined by crystal symmetry. An approximate (isotropic) treatment of cell esds is used for estimating esds involving l.s. planes.

Fractional atomic coordinates and isotropic or equivalent isotropic displacement parameters $\left(\AA^{2}\right)$

\begin{tabular}{llllll}
\hline & $x$ & $y$ & $z$ & $U_{\text {iso }} / U_{\text {eq }}$ & Occ. $(<1)$ \\
\hline Cu1 & $0.64047(12)$ & $0.74631(16)$ & $0.76709(10)$ & $0.0112(2)$ & \\
Cu2 & $0.40646(10)$ & $0.48699(12)$ & $0.51341(8)$ & $0.0113(4)$ & \\
Cu3 & $0.37581(9)$ & $0.98271(12)$ & $0.52445(7)$ & $0.0117(4)$ & \\
Cu4 & $0.14420(12)$ & $0.75064(16)$ & $0.26663(9)$ & $0.0108(2)$ & \\
O1 & $0.4814(10)$ & $0.4981(7)$ & $0.7249(8)$ & $0.0150(14)$ & \\
H1 & $0.398(10)$ & $0.481(12)$ & $0.765(10)$ & $0.022^{*}$ & \\
O2 & $0.5487(8)$ & $0.2232(8)$ & $0.5561(7)$ & $0.0129(11)$ & \\
H2 & $0.625(9)$ & $0.211(12)$ & $0.502(8)$ & $0.019^{*}$ & \\
O3 & $0.4523(10)$ & $0.9538(8)$ & $0.7302(7)$ & $0.0157(12)$ & \\
H3 & $0.382(10)$ & $0.941(14)$ & $0.779(8)$ & $0.024^{*}$ & \\
O4 & $0.3320(10)$ & $0.4581(8)$ & $0.3087(7)$ & $0.0132(12)$ & \\
H4 & $0.408(10)$ & $0.436(12)$ & $0.263(8)$ & $0.020^{*}$ & \\
O5 & $0.2300(9)$ & $0.7197(7)$ & $0.4785(7)$ & $0.0145(11)$ & \\
H5 & $0.134(9)$ & $0.715(12)$ & $0.505(9)$ & $0.022^{*}$ & \\
O6 & $0.3032(10)$ & $1.0043(7)$ & $0.3128(7)$ & $0.0124(13)$ & \\
H6 & $0.376(10)$ & $1.021(11)$ & $0.262(9)$ & $0.019^{*}$ & \\
O11 & $0.6098(9)$ & $0.7456(7)$ & $0.5254(8)$ & $0.0170(12)^{*}$ & \\
O12 & $0.7217(8)$ & $0.6493(7)$ & $0.3281(6)$ & $0.0221(10)^{*}$ & \\
S1A & $0.7607(9)$ & $0.7811(8)$ & $0.4618(7)$ & $0.0195(19)^{*}$ & 0.7 \\
O13A & $0.7641(12)$ & $1.0103(9)$ & $0.4267(9)$ & $0.0178(16)^{*}$ & 0.7 \\
O14A & $0.9297(11)$ & $0.7171(13)$ & $0.5649(9)$ & $0.0204(17)^{*}$ & 0.7 \\
S1B & $0.7426(14)$ & $0.7916(17)$ & $0.4462(11)$ & $0.009(3)^{*}$ & 0.3 \\
O13B & $0.699(2)$ & $1.008(2)$ & $0.3789(19)$ & $0.019(4)^{*}$ & 0.3 \\
O14B & $0.922(2)$ & $0.799(3)$ & $0.5522(17)$ & $0.018(4)^{*}$ & 0.3 \\
O7 & $0.6288(8)$ & $0.7998(8)$ & $0.0410(7)$ & $0.0207(10)$ & \\
H7B & $0.645(10)$ & $0.722(10)$ & $0.117(6)$ & $0.031^{*}$ & \\
H7A & $0.526(6)$ & $0.869(11)$ & $0.020(8)$ & $0.031^{*}$ & \\
& & &
\end{tabular}

Atomic displacement parameters $\left(\AA^{2}\right)$

\begin{tabular}{lllllll}
\hline & $U^{11}$ & $U^{22}$ & $U^{33}$ & $U^{12}$ & $U^{13}$ & $U^{23}$ \\
\hline $\mathrm{Cu} 1$ & $0.0116(4)$ & $0.0092(4)$ & $0.0113(4)$ & $0.0003(2)$ & $0.0010(3)$ & $0.0002(2)$ \\
$\mathrm{Cu} 2$ & $0.0131(9)$ & $0.0097(4)$ & $0.0097(6)$ & $0.0000(4)$ & $0.0012(5)$ & $0.0004(4)$ \\
$\mathrm{Cu} 3$ & $0.0124(9)$ & $0.0108(4)$ & $0.0098(6)$ & $0.0000(5)$ & $0.0002(5)$ & $-0.0005(5)$
\end{tabular}




\begin{tabular}{lllllll}
$\mathrm{Cu} 4$ & $0.0124(4)$ & $0.0094(4)$ & $0.0094(4)$ & $-0.0011(2)$ & $0.0011(3)$ & $0.0001(2)$ \\
$\mathrm{O} 1$ & $0.016(4)$ & $0.016(2)$ & $0.013(3)$ & $-0.0010(19)$ & $0.005(2)$ & $0.0006(16)$ \\
$\mathrm{O} 2$ & $0.013(3)$ & $0.014(3)$ & $0.011(2)$ & $0.001(2)$ & $0.002(2)$ & $-0.0001(18)$ \\
$\mathrm{O} 3$ & $0.017(3)$ & $0.018(3)$ & $0.013(3)$ & $0.000(2)$ & $0.006(2)$ & $0.000(2)$ \\
$\mathrm{O} 4$ & $0.015(3)$ & $0.014(3)$ & $0.010(3)$ & $0.001(2)$ & $0.003(2)$ & $-0.001(2)$ \\
$\mathrm{O} 5$ & $0.016(3)$ & $0.015(2)$ & $0.014(2)$ & $-0.001(2)$ & $0.006(2)$ & $0.000(2)$ \\
O6 & $0.010(3)$ & $0.014(3)$ & $0.013(3)$ & $-0.0005(18)$ & $0.003(2)$ & $0.0006(17)$ \\
O7 & $0.018(2)$ & $0.021(3)$ & $0.022(3)$ & $0.002(2)$ & $0.005(2)$ & $0.004(2)$ \\
\hline
\end{tabular}

Geometric parameters $\left(\AA,{ }^{\circ}\right)$

\begin{tabular}{|c|c|c|c|}
\hline $\mathrm{Cu} 1-\mathrm{O} 3$ & $1.929(7)$ & $\mathrm{Cu} 4-\mathrm{O} 5$ & $1.986(7)$ \\
\hline $\mathrm{Cu} 1-\mathrm{O} 4^{\mathrm{i}}$ & $1.933(7)$ & $\mathrm{Cu} 4-\mathrm{O}^{\mathrm{v}}$ & $1.993(6)$ \\
\hline $\mathrm{Cu} 1-\mathrm{O} 1$ & $1.974(6)$ & $\mathrm{Cu} 4-\mathrm{O} 6$ & $2.002(6)$ \\
\hline $\mathrm{Cu} 1-\mathrm{O}^{\mathrm{ii}}$ & $1.997(6)$ & $\mathrm{Cu} 4-\mathrm{O} 4$ & $2.327(6)$ \\
\hline $\mathrm{Cu} 1-\mathrm{O} 11$ & $2.300(7)$ & $\mathrm{Cu} 4-\mathrm{O}^{\mathrm{vi}}$ & $2.363(6)$ \\
\hline $\mathrm{Cu} 1-\mathrm{Cu} 3$ & $3.0399(14)$ & $\mathrm{O} 1-\mathrm{H} 1$ & $0.87(6)$ \\
\hline $\mathrm{Cu} 1-\mathrm{Cu} 2^{\mathrm{i}}$ & $3.0558(14)$ & $\mathrm{O} 2-\mathrm{H} 2$ & $0.91(6)$ \\
\hline $\mathrm{Cu} 2-\mathrm{O} 4$ & $1.918(7)$ & $\mathrm{O} 3-\mathrm{H} 3$ & $0.83(6)$ \\
\hline $\mathrm{Cu} 2-\mathrm{O} 1$ & $1.974(7)$ & $\mathrm{O} 4-\mathrm{H} 4$ & $0.86(6)$ \\
\hline $\mathrm{Cu} 2-\mathrm{O} 5$ & $1.981(6)$ & $\mathrm{O} 5-\mathrm{H} 5$ & $0.87(6)$ \\
\hline $\mathrm{Cu} 2-\mathrm{O} 2$ & $1.984(5)$ & O6-H6 & $0.86(6)$ \\
\hline $\mathrm{Cu} 2-\mathrm{O} 11$ & $2.265(6)$ & $\mathrm{O} 11-\mathrm{S} 1 \mathrm{~B}$ & $1.499(11)$ \\
\hline $\mathrm{Cu} 2-\mathrm{Cu} 4^{\mathrm{i}}$ & $3.0194(14)$ & $\mathrm{O} 11-\mathrm{S} 1 \mathrm{~A}$ & $1.508(8)$ \\
\hline $\mathrm{Cu} 3-\mathrm{O} 3$ & $1.927(7)$ & $\mathrm{O} 12-\mathrm{S} 1 \mathrm{~B}$ & $1.435(11)$ \\
\hline $\mathrm{Cu} 3-\mathrm{O} 6$ & $1.979(7)$ & $\mathrm{O} 12-\mathrm{S} 1 \mathrm{~A}$ & $1.503(6)$ \\
\hline $\mathrm{Cu} 3-\mathrm{O} 5$ & $1.996(6)$ & $\mathrm{S} 1 \mathrm{~A}-\mathrm{O} 14 \mathrm{~A}$ & $1.463(9)$ \\
\hline $\mathrm{Cu} 3-\mathrm{O} 2^{\mathrm{iii}}$ & $2.002(6)$ & $\mathrm{S} 1 \mathrm{~A}-\mathrm{O} 13 \mathrm{~A}$ & $1.495(8)$ \\
\hline $\mathrm{Cu} 3-\mathrm{O} 11$ & $2.370(6)$ & $\mathrm{S} 1 \mathrm{~B}-\mathrm{O} 14 \mathrm{~B}$ & $1.479(17)$ \\
\hline $\mathrm{Cu} 3-\mathrm{O}^{\mathrm{iv}}$ & $2.422(6)$ & $\mathrm{S} 1 \mathrm{~B}-\mathrm{O} 13 \mathrm{~B}$ & $1.516(19)$ \\
\hline $\mathrm{Cu} 3-\mathrm{Cu} 4$ & $3.0135(14)$ & $\mathrm{O} 7-\mathrm{H} 7 \mathrm{~B}$ & $0.87(3)$ \\
\hline $\mathrm{Cu} 4-\mathrm{O} 2^{\mathrm{v}}$ & $1.975(7)$ & $\mathrm{O} 7-\mathrm{H} 7 \mathrm{~A}$ & $0.89(3)$ \\
\hline $\mathrm{O} 3-\mathrm{Cu} 1-\mathrm{O} 4^{\mathrm{i}}$ & $178.6(4)$ & $\mathrm{O} 6-\mathrm{Cu} 4-\mathrm{O} 4$ & $106.4(3)$ \\
\hline $\mathrm{O} 3-\mathrm{Cu} 1-\mathrm{O} 1$ & $96.0(3)$ & $\mathrm{O} 2^{\mathrm{v}}-\mathrm{Cu} 4-\mathrm{O}^{\mathrm{vi}}$ & $75.5(2)$ \\
\hline $\mathrm{O} 4-\mathrm{Cu} 1-\mathrm{O} 1$ & $85.0(3)$ & $\mathrm{O} 5-\mathrm{Cu} 4-\mathrm{O}^{\mathrm{vi}}$ & $103.8(2)$ \\
\hline $\mathrm{O} 3-\mathrm{Cu} 1-\mathrm{O}^{\mathrm{ii}}$ & $84.5(3)$ & $\mathrm{O} 1^{\mathrm{v}}-\mathrm{Cu} 4-\mathrm{O} 3^{\mathrm{vi}}$ & $104.9(3)$ \\
\hline $\mathrm{O} 4-\mathrm{Cu} 1-\mathrm{O}^{\mathrm{ii}}$ & $94.5(3)$ & $\mathrm{O} 6-\mathrm{Cu} 4-\mathrm{O}^{\mathrm{vi}}$ & $73.9(2)$ \\
\hline $\mathrm{O} 1-\mathrm{Cu} 1-\mathrm{O}^{\mathrm{ii}}$ & $179.1(4)$ & $\mathrm{O} 4-\mathrm{Cu} 4-\mathrm{O}^{\mathrm{vi}}$ & $178.4(3)$ \\
\hline $\mathrm{O} 3-\mathrm{Cu} 1-\mathrm{O} 11$ & $88.2(3)$ & $\mathrm{O} 2^{\mathrm{v}}-\mathrm{Cu} 4-\mathrm{Cu} 3$ & $140.94(16)$ \\
\hline $\mathrm{O} 4-\mathrm{Cu} 1-\mathrm{O} 11$ & $93.0(2)$ & $\mathrm{O} 5-\mathrm{Cu} 4-\mathrm{Cu} 3$ & $40.93(15)$ \\
\hline $\mathrm{O} 1-\mathrm{Cu} 1-\mathrm{O} 11$ & $85.3(3)$ & $\mathrm{O} 1^{v}-\mathrm{Cu} 4-\mathrm{Cu} 3$ & $138.3(2)$ \\
\hline $\mathrm{O}^{\mathrm{ii}}-\mathrm{Cu} 1-\mathrm{O} 11$ & $95.5(2)$ & $\mathrm{O} 6-\mathrm{Cu} 4-\mathrm{Cu} 3$ & $40.5(2)$ \\
\hline $\mathrm{O} 3-\mathrm{Cu} 1-\mathrm{Cu} 3$ & $37.9(2)$ & $\mathrm{O} 4-\mathrm{Cu} 4-\mathrm{Cu} 3$ & $92.84(17)$ \\
\hline $\mathrm{O} 4-\mathrm{Cu} 1-\mathrm{Cu} 3$ & $143.3(2)$ & $\mathrm{O} 3^{\mathrm{vi}}-\mathrm{Cu} 4-\mathrm{Cu} 3$ & $86.39(18)$ \\
\hline $\mathrm{O} 1-\mathrm{Cu} 1-\mathrm{Cu} 3$ & $89.3(2)$ & $\mathrm{O} 2^{\mathrm{v}}-\mathrm{Cu} 4-\mathrm{Cu} 2^{\mathrm{v}}$ & $40.42(15)$ \\
\hline $\mathrm{O} 66^{\mathrm{ii}}-\mathrm{Cu} 1-\mathrm{Cu} 3$ & $91.51(19)$ & $\mathrm{O} 5-\mathrm{Cu} 4-\mathrm{Cu} 2^{\mathrm{v}}$ & $137.68(16)$ \\
\hline $\mathrm{O} 11-\mathrm{Cu} 1-\mathrm{Cu} 3$ & $50.39(16)$ & $\mathrm{O} 1^{\mathrm{v}}-\mathrm{Cu} 4-\mathrm{Cu} 2^{\mathrm{v}}$ & $40.2(2)$ \\
\hline
\end{tabular}




\begin{tabular}{|c|c|c|c|}
\hline $\mathrm{O} 3-\mathrm{Cu} 1-\mathrm{Cu}^{2}$ & $141.5(2)$ & $\mathrm{O} 6-\mathrm{Cu} 4-\mathrm{Cu} 2^{\mathrm{v}}$ & $140.9(2)$ \\
\hline $\mathrm{O} 4-\mathrm{Cu} 1-\mathrm{Cu}^{\mathrm{i}}$ & $37.3(2)$ & $\mathrm{O} 4-\mathrm{Cu} 4-\mathrm{Cu} 2^{\mathrm{v}}$ & $87.18(17)$ \\
\hline $\mathrm{O} 1-\mathrm{Cu} 1-\mathrm{Cu} 2^{\mathrm{i}}$ & $91.1(2)$ & $\mathrm{O} 3^{\mathrm{vi}}-\mathrm{Cu} 4-\mathrm{Cu} 2^{\mathrm{v}}$ & $93.56(17)$ \\
\hline $\mathrm{O} 6^{\mathrm{ii}}-\mathrm{Cu} 1-\mathrm{Cu} 2^{\mathrm{i}}$ & 88.07 (19) & $\mathrm{Cu} 3-\mathrm{Cu} 4-\mathrm{Cu} 2^{\mathrm{v}}$ & $178.43(5)$ \\
\hline $\mathrm{O} 11-\mathrm{Cu} 1-\mathrm{Cu} 2^{\mathrm{i}}$ & $130.19(16)$ & $\mathrm{Cu} 1-\mathrm{O} 1-\mathrm{Cu} 2$ & $102.7(3)$ \\
\hline $\mathrm{Cu} 3-\mathrm{Cu} 1-\mathrm{Cu} 2^{\mathrm{i}}$ & $179.32(4)$ & $\mathrm{Cu} 1-\mathrm{O} 1-\mathrm{Cu} 4^{\mathrm{i}}$ & $105.2(4)$ \\
\hline $\mathrm{O} 4-\mathrm{Cu} 2-\mathrm{O} 1$ & $176.5(2)$ & $\mathrm{Cu} 2-\mathrm{O} 1-\mathrm{Cu} 4^{\mathrm{i}}$ & $99.1(3)$ \\
\hline $\mathrm{O} 4-\mathrm{Cu} 2-\mathrm{O} 5$ & $84.9(3)$ & $\mathrm{Cu} 1-\mathrm{O} 1-\mathrm{H} 1$ & $122(5)$ \\
\hline $\mathrm{O} 1-\mathrm{Cu} 2-\mathrm{O} 5$ & $97.4(3)$ & $\mathrm{Cu} 2-\mathrm{O} 1-\mathrm{H} 1$ & $116(6)$ \\
\hline $\mathrm{O} 4-\mathrm{Cu} 2-\mathrm{O} 2$ & $96.7(2)$ & $\mathrm{Cu} 4-\mathrm{O} 1-\mathrm{H} 1$ & $110(5)$ \\
\hline $\mathrm{O} 1-\mathrm{Cu} 2-\mathrm{O} 2$ & $80.6(3)$ & $\mathrm{Cu} 4-\mathrm{O} 2-\mathrm{Cu} 2$ & $99.4(2)$ \\
\hline $\mathrm{O} 5-\mathrm{Cu} 2-\mathrm{O} 2$ & $169.6(3)$ & $\mathrm{Cu} 4^{\mathrm{i}}-\mathrm{O} 2-\mathrm{Cu} 3^{\mathrm{vii}}$ & $104.7(3)$ \\
\hline $\mathrm{O} 4-\mathrm{Cu} 2-\mathrm{O} 11$ & $96.6(2)$ & $\mathrm{Cu} 2-\mathrm{O} 2-\mathrm{Cu}^{\mathrm{vii}}$ & $107.3(3)$ \\
\hline $\mathrm{O} 1-\mathrm{Cu} 2-\mathrm{O} 11$ & $86.2(3)$ & $\mathrm{Cu} 4-\mathrm{O} 2-\mathrm{H} 2$ & $120(5)$ \\
\hline $\mathrm{O} 5-\mathrm{Cu} 2-\mathrm{O} 11$ & $85.1(2)$ & $\mathrm{Cu} 2-\mathrm{O} 2-\mathrm{H} 2$ & $112(5)$ \\
\hline $\mathrm{O} 2-\mathrm{Cu} 2-\mathrm{O} 11$ & $104.8(3)$ & $\mathrm{Cu} 3^{\mathrm{vii}}-\mathrm{O} 2-\mathrm{H} 2$ & $112(5)$ \\
\hline $\mathrm{O} 4-\mathrm{Cu} 2-\mathrm{Cu} 4^{\mathrm{i}}$ & $136.77(17)$ & $\mathrm{Cu} 3-\mathrm{O} 3-\mathrm{Cu} 1$ & $104.1(3)$ \\
\hline $\mathrm{O} 1-\mathrm{Cu} 2-\mathrm{Cu} 4^{\mathrm{i}}$ & $40.67(17)$ & $\mathrm{Cu} 3-\mathrm{O} 3-\mathrm{Cu} 4^{\mathrm{ii}}$ & $93.9(2)$ \\
\hline $\mathrm{O} 5-\mathrm{Cu} 2-\mathrm{Cu} 4^{\mathrm{i}}$ & $137.9(2)$ & $\mathrm{Cu} 1-\mathrm{O} 3-\mathrm{Cu} 4^{\mathrm{ii}}$ & $95.5(3)$ \\
\hline $\mathrm{O} 2-\mathrm{Cu} 2-\mathrm{Cu} 4^{\mathrm{i}}$ & $40.18(19)$ & $\mathrm{Cu} 3-\mathrm{O} 3-\mathrm{H} 3$ & $124(6)$ \\
\hline $\mathrm{O} 11-\mathrm{Cu} 2-\mathrm{Cu} 4^{\mathrm{i}}$ & $93.75(18)$ & $\mathrm{Cu} 1-\mathrm{O} 3-\mathrm{H} 3$ & $115(6)$ \\
\hline $\mathrm{O} 4-\mathrm{Cu} 2-\mathrm{Cu}^{v}$ & $37.7(2)$ & $\mathrm{Cu} 44^{\mathrm{ii}}-\mathrm{O} 3-\mathrm{H} 3$ & $119(6)$ \\
\hline $\mathrm{O} 1-\mathrm{Cu} 2-\mathrm{Cu}^{\mathrm{v}}$ & $139.7(2)$ & $\mathrm{Cu} 2-\mathrm{O} 4-\mathrm{Cu}^{\mathrm{v}}$ & $105.0(3)$ \\
\hline $\mathrm{O} 5-\mathrm{Cu} 2-\mathrm{Cu} 1^{v}$ & $86.97(19)$ & $\mathrm{Cu} 2-\mathrm{O} 4-\mathrm{Cu} 4$ & $95.1(2)$ \\
\hline $\mathrm{O} 2-\mathrm{Cu} 2-\mathrm{Cu}^{\mathrm{v}}$ & $88.10(19)$ & $\mathrm{Cu} 1^{\mathrm{v}}-\mathrm{O} 4-\mathrm{Cu} 4$ & $94.9(3)$ \\
\hline $\mathrm{O} 11-\mathrm{Cu} 2-\mathrm{Cu}^{\mathrm{v}}$ & $134.10(19)$ & $\mathrm{Cu} 2-\mathrm{O} 4-\mathrm{H} 4$ & $121(5)$ \\
\hline $\mathrm{Cu} 4-\mathrm{Cu} 2-\mathrm{Cu}^{\mathrm{i}}$ & $120.91(4)$ & $\mathrm{Cu} 1^{\mathrm{v}}-\mathrm{O} 4-\mathrm{H} 4$ & $113(5)$ \\
\hline $\mathrm{O} 3-\mathrm{Cu} 3-\mathrm{O} 6$ & $178.0(3)$ & $\mathrm{Cu} 4-\mathrm{O} 4-\mathrm{H} 4$ & $123(5)$ \\
\hline $\mathrm{O} 3-\mathrm{Cu} 3-\mathrm{O} 5$ & $97.7(2)$ & $\mathrm{Cu} 2-\mathrm{O} 5-\mathrm{Cu} 4$ & $104.8(3)$ \\
\hline $\mathrm{O} 6-\mathrm{Cu} 3-\mathrm{O} 5$ & $81.7(3)$ & $\mathrm{Cu} 2-\mathrm{O} 5-\mathrm{Cu} 3$ & $105.1(3)$ \\
\hline $\mathrm{O} 3-\mathrm{Cu} 3-\mathrm{O} 2^{\mathrm{iii}}$ & $85.7(3)$ & $\mathrm{Cu} 4-\mathrm{O} 5-\mathrm{Cu} 3$ & $98.4(2)$ \\
\hline $\mathrm{O} 6-\mathrm{Cu} 3-\mathrm{O} 2^{\mathrm{iii}}$ & $94.6(2)$ & $\mathrm{Cu} 2-\mathrm{O} 5-\mathrm{H} 5$ & $123(5)$ \\
\hline $\mathrm{O} 5-\mathrm{Cu} 3-\mathrm{O} 2^{\mathrm{iii}}$ & $170.8(3)$ & $\mathrm{Cu} 4-\mathrm{O} 5-\mathrm{H} 5$ & $105(6)$ \\
\hline $\mathrm{O} 3-\mathrm{Cu} 3-\mathrm{O} 11$ & $86.3(3)$ & $\mathrm{Cu} 3-\mathrm{O} 5-\mathrm{H} 5$ & $117(5)$ \\
\hline $\mathrm{O} 6-\mathrm{Cu} 3-\mathrm{O} 11$ & $91.7(2)$ & $\mathrm{Cu} 3-\mathrm{O} 6-\mathrm{Cu} 1^{\mathrm{vi}}$ & $104.7(3)$ \\
\hline $\mathrm{O} 5-\mathrm{Cu} 3-\mathrm{O} 11$ & $82.0(2)$ & $\mathrm{Cu} 3-\mathrm{O} 6-\mathrm{Cu} 4$ & $98.4(3)$ \\
\hline $\mathrm{O} 22^{\mathrm{iii}}-\mathrm{Cu} 3-\mathrm{O} 11$ & $89.7(2)$ & $\mathrm{Cu} 1^{\mathrm{vi}}-\mathrm{O} 6-\mathrm{Cu} 4$ & $105.8(4)$ \\
\hline $\mathrm{O} 3-\mathrm{Cu} 3-\mathrm{O}^{\mathrm{iv}}$ & $89.5(2)$ & $\mathrm{Cu} 3-\mathrm{O} 6-\mathrm{H} 6$ & $125(6)$ \\
\hline $\mathrm{O} 6-\mathrm{Cu} 3-\mathrm{O}^{\mathrm{iv}}$ & $92.5(3)$ & $\mathrm{Cu}^{\mathrm{vi}}-\mathrm{O} 6-\mathrm{H} 6$ & $105(5)$ \\
\hline $\mathrm{O} 5-\mathrm{Cu} 3-\mathrm{O}^{\mathrm{iv}}$ & $94.3(2)$ & $\mathrm{Cu} 4-\mathrm{O} 6-\mathrm{H} 6$ & $116(5)$ \\
\hline $\mathrm{O} 2^{\mathrm{iii}}-\mathrm{Cu} 3-\mathrm{O} 7^{\mathrm{iv}}$ & $94.3(2)$ & $\mathrm{S} 1 \mathrm{~B}-\mathrm{O} 11-\mathrm{Cu} 2$ & $134.4(5)$ \\
\hline $\mathrm{O} 11-\mathrm{Cu} 3-\mathrm{O}^{\mathrm{iv}}$ & $174.0(2)$ & $\mathrm{S} 1 \mathrm{~A}-\mathrm{O} 11-\mathrm{Cu} 2$ & $135.5(4)$ \\
\hline $\mathrm{O} 3-\mathrm{Cu} 3-\mathrm{Cu} 4$ & $138.42(17)$ & $\mathrm{S} 1 \mathrm{~B}-\mathrm{O} 11-\mathrm{Cu} 1$ & $130.7(5)$ \\
\hline $\mathrm{O} 6-\mathrm{Cu} 3-\mathrm{Cu} 4$ & $41.10(16)$ & $\mathrm{S} 1 \mathrm{~A}-\mathrm{O} 11-\mathrm{Cu} 1$ & $124.6(4)$ \\
\hline $\mathrm{O} 5-\mathrm{Cu} 3-\mathrm{Cu} 4$ & $40.70(19)$ & $\mathrm{Cu} 2-\mathrm{O} 11-\mathrm{Cu} 1$ & $84.9(2)$ \\
\hline $\mathrm{O} 2^{\mathrm{iii}}-\mathrm{Cu} 3-\mathrm{Cu} 4$ & $135.46(18)$ & $\mathrm{S} 1 \mathrm{~B}-\mathrm{O} 11-\mathrm{Cu} 3$ & $122.0(5)$ \\
\hline $\mathrm{O} 11-\mathrm{Cu} 3-\mathrm{Cu} 4$ & 88.09 (17) & $\mathrm{S} 1 \mathrm{~A}-\mathrm{O} 11-\mathrm{Cu} 3$ & $127.2(3)$ \\
\hline $\mathrm{O}^{\mathrm{iv}}-\mathrm{Cu} 3-\mathrm{Cu} 4$ & $92.24(15)$ & $\mathrm{Cu} 2-\mathrm{O} 11-\mathrm{Cu} 3$ & $85.8(2)$ \\
\hline
\end{tabular}




$\begin{array}{llll}\mathrm{O} 3-\mathrm{Cu} 3-\mathrm{Cu} 1 & 37.98(19) & \mathrm{Cu} 1-\mathrm{O} 11-\mathrm{Cu} 3 & 81.2(2) \\ \mathrm{O} 6-\mathrm{Cu} 3-\mathrm{Cu} 1 & 139.99(19) & \mathrm{O} 14 \mathrm{~A}-\mathrm{S} 1 \mathrm{~A}-\mathrm{O} 13 \mathrm{~A} & 110.7(6) \\ \mathrm{O} 5-\mathrm{Cu} 3-\mathrm{Cu} 1 & 88.6(2) & \mathrm{O} 14 \mathrm{~A}-\mathrm{S} 1 \mathrm{~A}-\mathrm{O} 12 & 110.6(5) \\ \mathrm{O} 2^{\mathrm{iii}}-\mathrm{Cu} 3-\mathrm{Cu} 1 & 88.94(17) & \mathrm{O} 13 \mathrm{~A}-\mathrm{S} 1 \mathrm{~A}-\mathrm{O} 12 & 110.8(5) \\ \mathrm{O} 11-\mathrm{Cu} 3-\mathrm{Cu} 1 & 48.41(18) & \mathrm{O} 14 \mathrm{~A}-\mathrm{S} 1 \mathrm{~A}-\mathrm{O} 11 & 109.6(5) \\ \mathrm{O} 7^{\mathrm{iv}}-\mathrm{Cu} 3-\mathrm{Cu} 1 & 127.04(15) & \mathrm{O} 13 \mathrm{~A}-\mathrm{S} 1 \mathrm{~A}-\mathrm{O} 11 & 108.0(5) \\ \mathrm{Cu} 4-\mathrm{Cu} 3-\mathrm{Cu} 1 & 120.83(4) & \mathrm{O} 12-\mathrm{S} 1 \mathrm{~A}-\mathrm{O} 11 & 107.1(4) \\ \mathrm{O} 2^{\mathrm{v}}-\mathrm{Cu} 4-\mathrm{O} 5 & 177.5(3) & \mathrm{O} 12-\mathrm{S} 1 \mathrm{~B}-\mathrm{O} 14 \mathrm{~B} & 116.1(10) \\ \mathrm{O} 2^{\mathrm{v}}-\mathrm{Cu} 4-\mathrm{O} 1^{\mathrm{v}} & 80.3(3) & \mathrm{O} 12-\mathrm{S} 1 \mathrm{~B}-\mathrm{O} 11 & 111.2(7) \\ \mathrm{O} 5-\mathrm{Cu} 4-\mathrm{O} 1^{\mathrm{v}} & 97.6(3) & \mathrm{O} 14 \mathrm{~B}-\mathrm{S} 1 \mathrm{~B}-\mathrm{O} 11 & 107.9(9) \\ \mathrm{O} 2^{\mathrm{v}}-\mathrm{Cu} 4-\mathrm{O} 6 & 100.6(3) & \mathrm{O} 12-\mathrm{S} 1 \mathrm{~B}-\mathrm{O} 13 \mathrm{~B} & 105.3(9) \\ \mathrm{O} 5-\mathrm{Cu} 4-\mathrm{O} 6 & 81.3(3) & \mathrm{O} 14 \mathrm{~B}-\mathrm{S} 1 \mathrm{~B}-\mathrm{O} 13 \mathrm{~B} & 107.3(12) \\ \mathrm{O} 1^{\mathrm{v}}-\mathrm{Cu} 4-\mathrm{O} 6 & 178.1(4) & \mathrm{O} 11-\mathrm{S} 1 \mathrm{~B}-\mathrm{O} 13 \mathrm{~B} & 118(5) \\ \mathrm{O} 2^{\mathrm{v}}-\mathrm{Cu} 4-\mathrm{O} 4 & 105.9(2) & \mathrm{Cu} 3^{\text {viii }}-\mathrm{O} 7-\mathrm{H} 7 \mathrm{~B} & 113(5) \\ \mathrm{O} 5-\mathrm{Cu} 4-\mathrm{O} 4 & 74.8(2) & \mathrm{Cu} 3^{\text {viii-O}}-\mathrm{O}-\mathrm{H} 7 \mathrm{~A} & 112(6) \\ \mathrm{O} 1^{\mathrm{v}}-\mathrm{Cu} 4-\mathrm{O} 4 & 74.8(2) & \mathrm{H} 7 \mathrm{~B}-\mathrm{O} 7-\mathrm{H} 7 \mathrm{~A} & \end{array}$

Symmetry codes: (i) $x+1 / 2,-y+1, z+1 / 2$; (ii) $x+1 / 2,-y+2, z+1 / 2$; (iii) $x, y+1, z$; (iv) $x-1 / 2,-y+2, z+1 / 2$; (v) $x-1 / 2,-y+1, z-1 / 2$; (vi) $x-1 / 2,-y+2, z-1 / 2$; (vii) $x, y-1, z$; (viii) $x+1 / 2,-y+2, z-1 / 2$.

Hydrogen-bond geometry $\left(A,{ }^{\circ}\right)$

\begin{tabular}{lllll}
\hline$D-\mathrm{H} \cdots A$ & $D-\mathrm{H}$ & $\mathrm{H} \cdots A$ & $D \cdots A$ & $D-\mathrm{H} \cdots A$ \\
\hline $\mathrm{O} 1-\mathrm{H} 1 \cdots \mathrm{O} 12^{\mathrm{ix}}$ & $0.87(6)$ & $1.86(6)$ & $2.693(9)$ & $160(7)$ \\
$\mathrm{O} 7-\mathrm{H} 7 B \cdots \mathrm{O} 12$ & $0.87(3)$ & $2.02(4)$ & $2.844(7)$ & $158(7)$ \\
$\mathrm{O} 2-\mathrm{H} 2 \cdots \mathrm{O} 13 A^{\text {vii }}$ & $0.91(6)$ & $1.95(6)$ & $2.746(9)$ & $144(7)$ \\
$\mathrm{O} 2-\mathrm{H} 2 \cdots \mathrm{O} 13 B^{\text {vii }}$ & $0.91(6)$ & $1.96(7)$ & $2.731(18)$ & $141(7)$ \\
$\mathrm{O} 3-\mathrm{H} 3 \cdots \mathrm{O} 13 A^{\text {iv }}$ & $0.83(6)$ & $1.96(6)$ & $2.757(10)$ & $161(9)$ \\
$\mathrm{O} 3-\mathrm{H} 3 \cdots \mathrm{O} 13 B^{\text {iv }}$ & $0.83(6)$ & $1.99(7)$ & $2.799(19)$ & $164(9)$ \\
$\mathrm{O} 7-\mathrm{H} 7 A \cdots \mathrm{O} 13 A^{\text {vi }}$ & $0.89(3)$ & $2.13(3)$ & $2.995(10)$ & $167(7)$ \\
$\mathrm{O} 4-\mathrm{H} 4 \cdots \mathrm{O} 14 A^{\mathrm{v}}$ & $0.86(6)$ & $2.22(7)$ & $2.929(9)$ & $141(7)$ \\
$\mathrm{O} 4-\mathrm{H} 4 \cdots \mathrm{O} 14 B^{\mathrm{v}}$ & $0.86(6)$ & $2.57(7)$ & $3.239(18)$ & $136(7)$ \\
$\mathrm{O} 5-\mathrm{H} 5 \cdots \mathrm{O} 14 A^{\mathrm{x}}$ & $0.87(6)$ & $1.86(6)$ & $2.727(9)$ & $177(8)$ \\
$\mathrm{O} 5-\mathrm{H} 5 \cdots \mathrm{O} 14 B^{\mathrm{x}}$ & $0.87(6)$ & $1.93(6)$ & $2.763(17)$ & $162(7)$ \\
$\mathrm{O} 6-\mathrm{H} 6 \cdots \mathrm{O} 13 B$ & $0.86(6)$ & $2.45(8)$ & $2.98(2)$ & $120(7)$ \\
$\mathrm{O} 6-\mathrm{H} 6 \cdots \mathrm{O} 14 B^{\mathrm{vi}}$ & $0.86(6)$ & $2.47(7)$ & $3.211(17)$ & $145(7)$ \\
$\mathrm{O} 7-\mathrm{H} 7 A \cdots \mathrm{O} 13 B^{\mathrm{vi}}$ & $0.89(3)$ & $2.64(4)$ & $3.489(18)$ & $161(7)$ \\
$\mathrm{O} 7-\mathrm{H} 7 A \cdots \mathrm{O} 14 B^{\text {vi }}$ & $0.89(3)$ & $2.31(6)$ & $3.034(19)$ & $139(7)$ \\
& & & &
\end{tabular}

Symmetry codes: (iv) $x-1 / 2,-y+2, z+1 / 2$; (v) $x-1 / 2,-y+1, z-1 / 2$; (vi) $x-1 / 2,-y+2, z-1 / 2$; (vii) $x, y-1, z$; (ix) $x-1 / 2,-y+1, z+1 / 2$; (x) $x-1, y, z$. 